\title{
Numerical and experimental study on cerebral contusion under impact loading
}

\author{
S. Aomura ${ }^{1}$, S. Fujiwara ${ }^{2} \&$ A. Nishimura ${ }^{2}$ \\ ${ }^{1}$ Graduate School of Tokyo Metropolitan University, Tokyo, Japan \\ ${ }^{2}$ Yokohama City University, Department of Legal Med., \\ Yokohama, Japan
}

\begin{abstract}
The mechanism of brain contusion is investigated using a finite element analysis. Prior to the numerical calculation for a finite element human head model, the experimental study of an impact loading for a water-filled acrylic cylindrical container was carried out in order to observe the fundamental physical phenomenon and to confirm the validity of the finite element analysis in comparison with the both numerical and experimental results. After the stroke, the deflection of the acrylic container wall propagated in both sides of the stroke point, and large negative pressure was generated just in the opposite side of the stroke point when deflections from both sides met. The time taken until the deflection reached the opposite side was close to the natural period of the waterfilled container and the period of fluctuation of internal pressure was equal to the natural frequency of the water-filled container. In numerical analysis the amplitude of the pressure fluctuation was slightly bigger for the tied container wall-water interface condition than for slide condition, and the damping was slightly bigger for the slide condition than for the tied condition, although the numerical and experimental pressure responses resembled each other approximately. Next, the human head model was analysed by FEM and the result was compared with the result of the experimental study reported by Nahum and good agreement was obtained. Five more loading cases were investigated. The deflection of the skull gave a large effect on the fluctuation of the internal pressure also for both coup and conrecoup contusions. Although in the frontal, occipital and under occipital region strokes the intracranial pressure of the coup and the contrecoup side was almost the same, in the parietal and temporal region strokes the intracranial pressure in the coup side was bigger than the contrecoup side.
\end{abstract}

Keywords: cerebral contusion, coup and contrecoup, impact loading, FEM. 


\section{Introduction}

In various head injuries caused by outside impact loading, brain contusion and DAI are mainly taken up as a direct failure to cerebral parenchyma. In brain contusion, there are coup contusion which is caused in the impact side and a contrecoup contusion which is caused in the opposite side of impact [1]. It has been pointed out that the brain contusion is caused by the rapid pressure fluctuation transmitted to the brain surface through the CSF, because the damage of the brain surface is more remarkable than DAI [2,3]. Although the theory that the brain surface is destroyed by the cerebral parenchyma colliding in skull is supported by intuitive comprehensibility, the situation in which the skull collides with the cerebral parenchyma actually is not explained in detail. Although cavitation theory can be trusted in which a pressure gradient develops in the fluid of the brain by impact, and the pressure gradient causes a contrecoup negative pressure at a point opposite the impact and forms cavitation bubbles and the subsequent collapse of the bubbles causes the brain tissue damage, collapse of bubbles in a head has not been observed. The various theories which explain mechanism of the generation of brain contusion have been reported in the past, there however is no theory which can sufficiently explain the whole mechanism.

Although the numerical analysis by finite element method has accomplished large development and become powerful tool for researchers recently [4-7], it is still important to verify the analytical result by the experiment using the simple physical model which has an analogy on a fundamental structure with a human head [8].

In this study, an experimental study of impact loading of a simple physical model was performed for the purpose of verification of analytical result. A waterfilled acrylic cylindrical container was struck by an iron hammer and pressure fluctuation in the water was measured. The result was compared with the FEM result and the followings were indicated. The arrival time of the first peak of pressure fluctuation at the opposite side of the stroke is later 10 times than the arrival time of the initial stress wave and it is almost equal to the natural period of vibration which transitionally continues in the container afterwards. And the rapid change of the internal pressure at the opposite side is caused by the deflection of a container wall.

Once validated, a computer model can help us understand injury mechanisms. First the finite element analysis was carried out for a human head model which consists of skull, CSF and brain. The loading condition equal to the experiment of Nahum et al [9] was given at the frontal region upper part and the both results showed good agreement. In addition, the calculation was carried out for five more loading cases to the frontal region center, the parietal region, the occipital region, the occipital under region and temporal region. In either stroke, coup and contrecoup contusion were observed. In the stroke of the frontal region, the occipital upper and under part, intensity of coup and contrecoup contusion were almost the same, while in the stroke of the parietal and temporal region, coup was superior than contrecoup contusion. Through the study, the finite element code LS-Dyna made by LSTC Co. was used for analysis. 


\section{Impact loading on a water-filled acrylic container}

The equipment of a stroke experiment of a water-filled acrylic cylindrical container is shown in Figure 1. The cylindrical container is $180 \mathrm{~mm}$ in diameter, $210 \mathrm{~mm}$ in height and $5 \mathrm{~mm}$ in thickness and sealed by putting an O-ring in so that the water may not leak out, and a lid is tightened up by 8 bolts. The container is fixed on a supply car which moves freely on the floor. The pressure was measured at the opposite side of stroke.

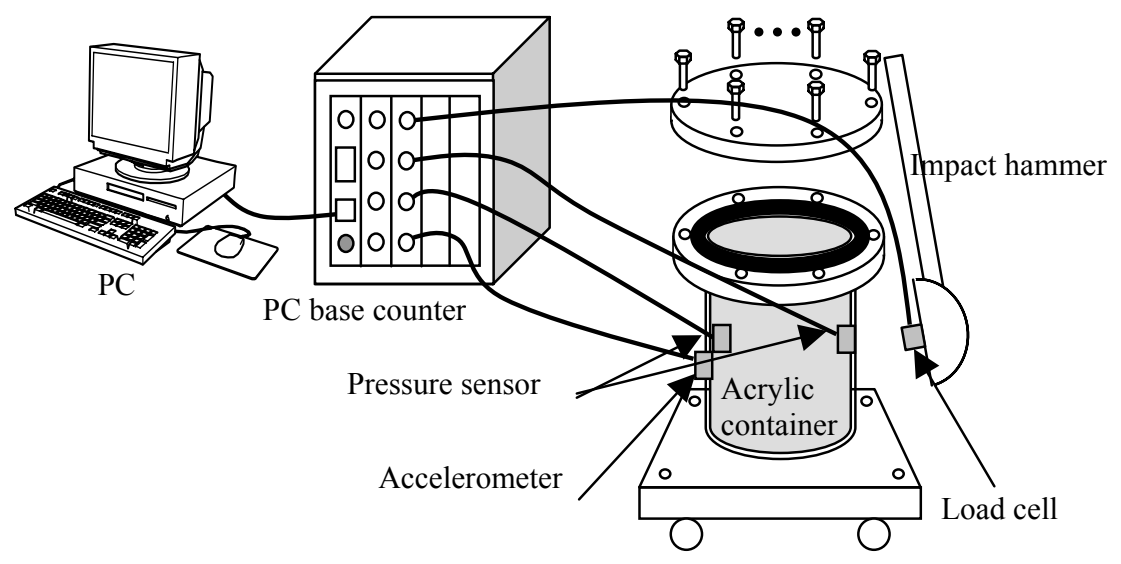

Figure 1: The equipment of a stroke experiment.

The finite element analysis of a container struck by a hammer was also carried out for two interface conditions of tied condition and slide condition between water and container wall. Results for both interface conditions are compared with experimental result, respectively, in Figure 2. In both experiment and finite element analyses, the sudden drops in negative pressure is observed just after the stroke and pressure fluctuation continues for a while. The maximum amplitude of the negative peak was calculated bigger by tied condition than by slide condition, and damping of pressure fluctuation is considered to be bigger by slide condition than by tied condition because all nodes are connected tightly and whole system becomes stiff in tied condition, however in slide condition relative displacement between two contact faces is allowed in the direction of contact face and the system is slightly flexible than tied case. Overall response of both interface conditions behave similarly and a good agreement is obtained between the results of FEM and experiment.

The frequency of the vibration which transitionally arises after the stroke in the container is approximately close to the natural frequency of the water-filled container. In Figure 3, the result of experimental frequency analysis of the waterfilled acrylic container is shown. The first natural frequency is around $315 \mathrm{~Hz}$, and the second frequency is around $420 \mathrm{~Hz}$. The frequency of the transient pressure fluctuation is around $400 \mathrm{~Hz}$. It takes theoretically $0.1 \sim 0.2 \mathrm{msec}$ that the 

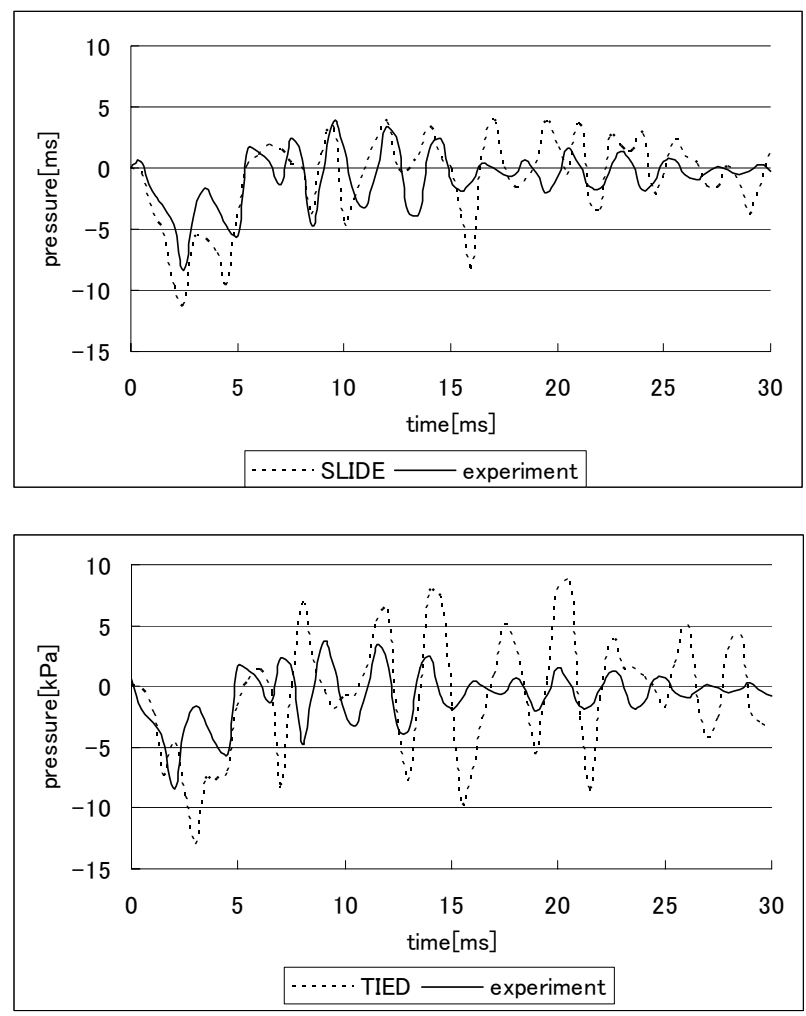

Figure 2: The comparison of pressure response obtained by experiment and FEM by slide condition (upper) and tied condition (lower). The pressure is measured at the opposite side of stroke.

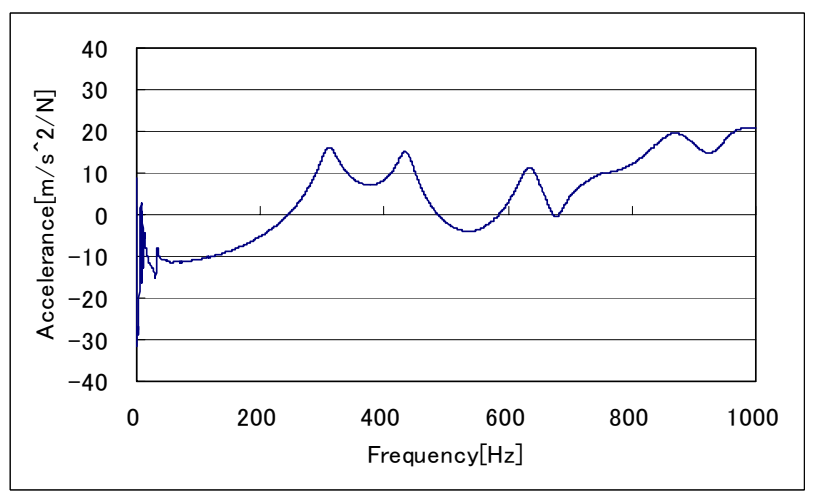

Figure 3: Natural frequency of a water-filled acrylic cylindrical container. 
stress wave propagates from stroke point to the opposite side through the acrylic container wall. It is observed that the frequency of fluctuation of internal pressure is considerably close to the natural frequency of the water-filled container.

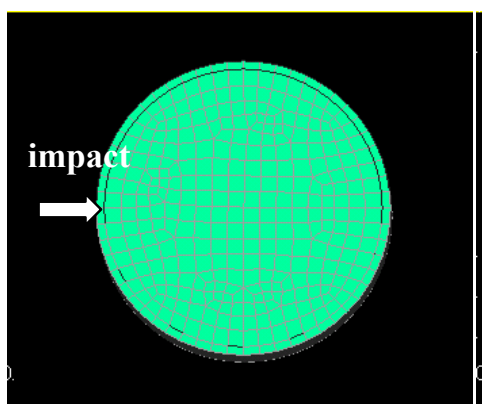

$0 \mathrm{~ms}$

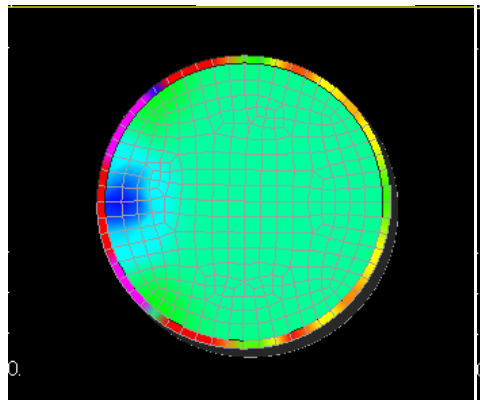

$0.5 \mathrm{~ms}$

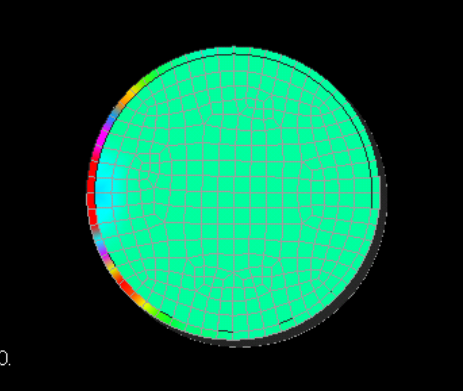

$0.1 \mathrm{~ms}$

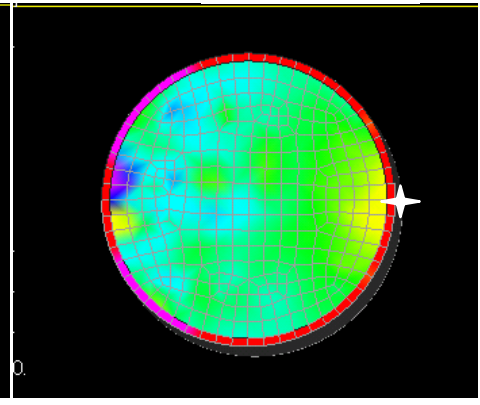

$2.3 \mathrm{~ms}$

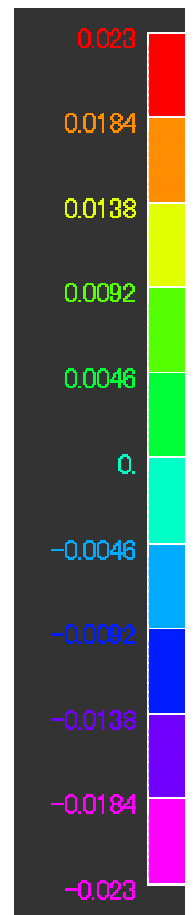

Figure 4: Generation of the sudden negative pressure at the opposite side of the stroke is shown $(2.3 \mathrm{~ms})$. Deflection of the container wall plays the important role to cause the sudden negative pressure change.

The horizontal section of the cylindrical container with water and transient stress and pressure distribution are shown in Figure 4. The reason that sudden negative pressure is caused at the opposite side of stroke by transmitting stress wave is considered as follows. The major stress wave with rather large deflection of container wall after the stroke transmits inside the acrylic wall at $0.1 \mathrm{msec}$ and $0.5 \mathrm{msec}$ after stroke is observed in Figure 4. This wave reaches the opposite side after $2 \mathrm{msec}$ almost, and then the first negative peak of pressure fluctuation is generated suddenly. This is much later than the theoretical arrival time of the stress wave through the acrylic wall. The large deflection of the container wall which caused the sudden negative pressure mentioned above was considered to be the first wave of the flexural vibration formed by stress waves going and back multiple times. Until the stress wave reached at the opposite side along the wall and caused the sudden negative pressure, there were no traces where the pressure 
wave propagated inside the water. The positive pressure which arose at the stroke side diffused and faded away without transmitting to the opposite side.

\section{Finite element analysis for a human head model}

\subsection{Analysis based on Nahum's condition}

Finite element analysis of a human head model was carried out. The head model consists of skull, CSF, and brain was made using the image of MRI for CSF and brain, and CT for skull. The geometric outline of the head model, the place and the direction of impact loading and measurement point are shown in Figure 5. Material properties of each part of the model by Galford [10] are shown in Table 1. To begin with the load was applied horizontally on the frontal bone in the mid-sagittal plane in an anterior-posterior direction. The skull was rotated forward so that the Frankfort anatomical plane was inclined $45^{\circ}$ to the horizontal, and this is the equal condition to the cadaver experiment carried out by Nahum [9], and the pressure response of the occiput was calculated. In numerical calculation, the same $5 \mathrm{~kg}$ iron impactor colliding with $6 \mathrm{~m} / \mathrm{s}$ used by Nahum could not be realized since various padding materials were interposed between the skull and impactor to vary the duration of the applied load. The time-force history given by Nahum was used. The load was applied on $846 \mathrm{~mm}^{2}$ area which was the same area given by Nahum. The free support condition for the head was adopted and this seems to be appropriate because the cadaver subjects were seated and were not fixed around the neck. Time history of input loading and experimental pressure response given by Nahum and pressure response calculated by FEM in this study are shown in Figure 6. Though the amplitude calculated is slightly bigger and damping of the fluctuation shown is smaller than the Nahum's experiment since it is calculated under the tied interface condition, both responses agree approximately.

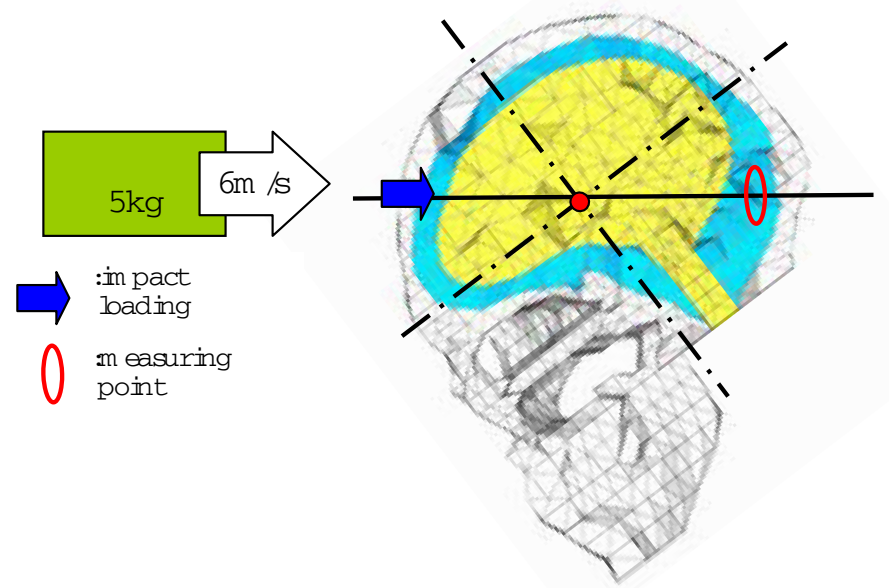

Figure 5: Finite element human head model and the direction of impact loading. 
Table 1: $\quad$ Material properties of skull, CSF and brain.

\begin{tabular}{c|c|c|c|c|c|c|c|c|c} 
& $\begin{array}{c}\rho \\
\left(\mathrm{kg} / \mathrm{m}^{3}\right)\end{array}$ & $\begin{array}{c}\mathrm{K} \\
(M P a)\end{array}$ & $\begin{array}{c}\mathrm{G} \\
(M P a)\end{array}$ & $\begin{array}{c}\mathrm{G}_{\mathrm{o}} \\
(M P a\end{array}$ & $\begin{array}{c}\mathrm{G}^{\infty} \\
(M P a)\end{array}$ & $\begin{array}{c}\beta \\
\left(s^{-1}\right)\end{array}$ & $\begin{array}{c}\sigma_{\mathrm{v}} \\
(M P a)\end{array}$ & $\begin{array}{c}\mathrm{E}_{\mathrm{t}} \\
(M P a)\end{array}$ & $\begin{array}{c}\mu \\
(M P a \cdot s)\end{array}$ \\
\hline Skull & 1456 & 7120 & 3470 & - & - & - & 41.8 & 4620 & - \\
\hline CSF & 998 & 2060 & - & - & - & - & - & - & $1.00 \mathrm{E}-09$ \\
\hline Brain & 1040 & 2190 & - & 0.528 & 0.5 & 5 & - & - & -
\end{tabular}

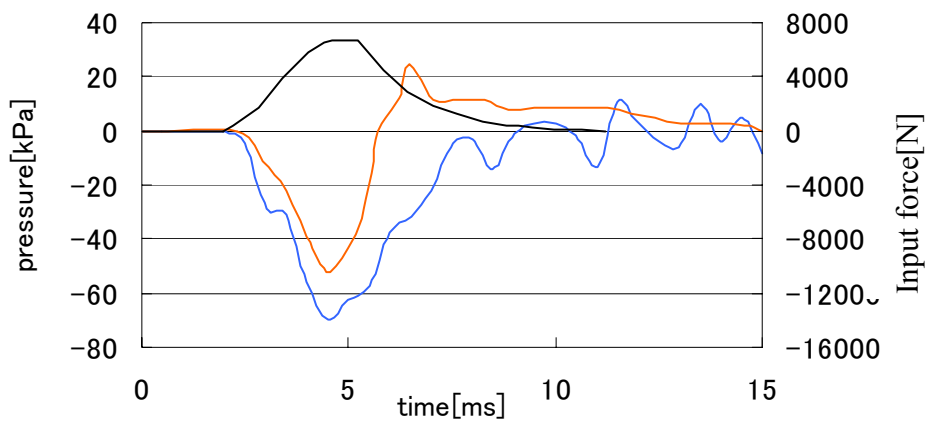

- Viscoelasticity(tied) — experiment — input force

Figure 6: Occipital intracranial pressure fluctuation obtained by experiment of Nahum [9] and obtained by FEM in this study, and time-history of impact loading at frontal bone.

\subsection{Impact loadings on several regions of head and pressure responses}

It is known by pathological examples that the relation of the pressure response in the stroke side and the opposite side of stroke depends on the percussion location. Then pressure responses in the stroke side and the opposite side of stroke were calculated by giving the same impact loading on the following five more regions of the head as shown in Figure 7; frontal region front face(1), parietal region (2), occiput (3), occipital under region (4) and temporal region (5). The direction of the stroke was set so as to pass through the center of gravity of a head in either case. The pressure response in stroke side and the opposite side for each impact loading is shown in Figure 7. Except for the parietal and temporal stroke, concerning the pressure in coup and contrecoup side, the sign was different and the amplitude was almost the same in any case. In parietal and temporal stroke, the pressure in the contrecoup side was smaller than the coup side.

In Figure 8, the stress distribution of skull and pressure distribution in brain at $3 \mathrm{msec}$ after parietal stroke(2) and at $4.5 \mathrm{msec}$ after occipital stroke(3) are shown. 
(1)
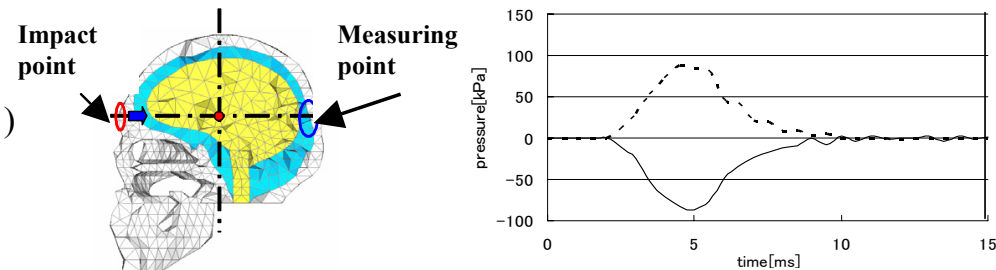

(2)

(3)
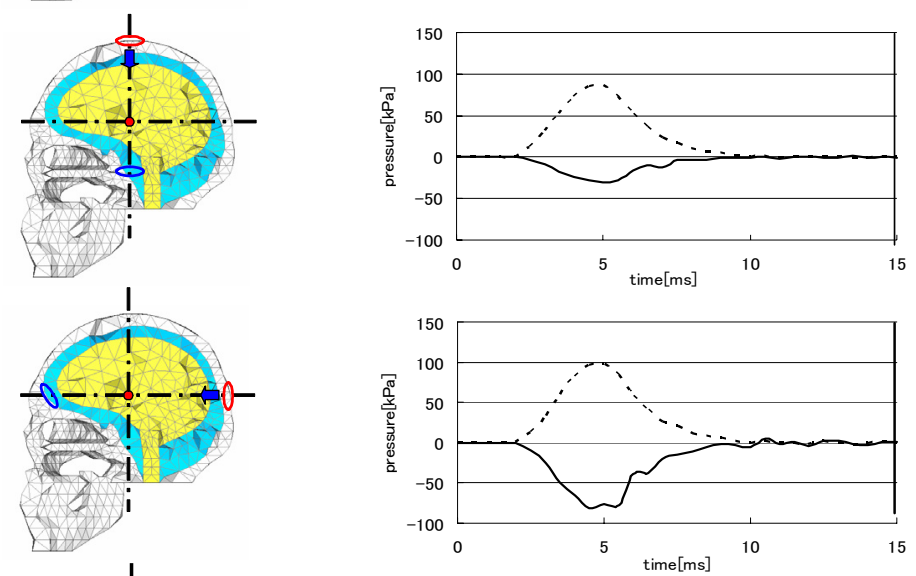

(4)
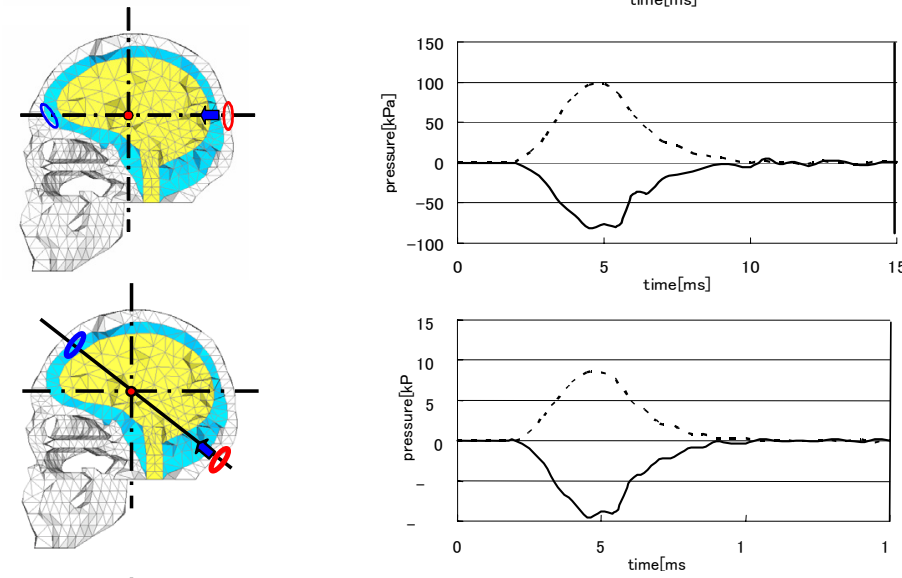

(5)
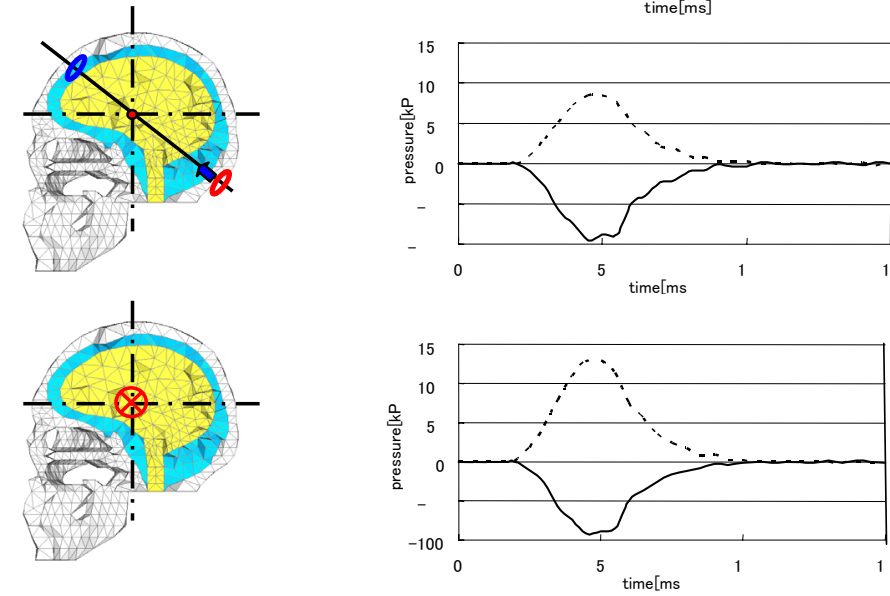

Impact (coup) side

Opposite(contrecoup) side

Figure 7: Intracranial pressure response of coup and contrecoup cases on several impact points. 


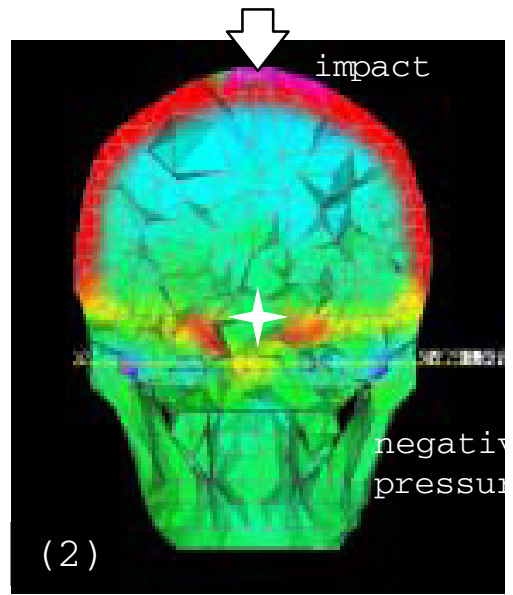

$3 \mathrm{~ms}$

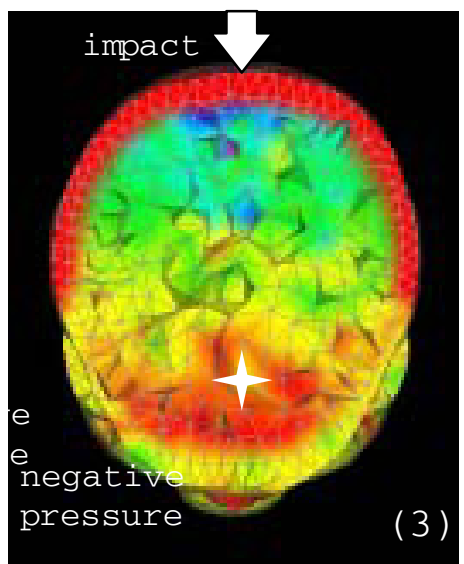

$4.5 \mathrm{~ms}$

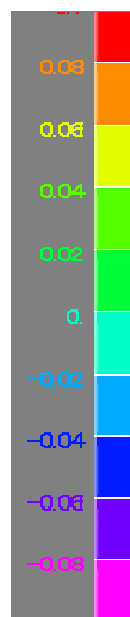

Figure 8: Stroke on parietal region (2) in which coup contusion is dominant, and occipital stroke (3) in which contrecoup is remarkable.

In occipital stroke in case (3), the high stress distribution of the skull is observed around the brain but the stress is not observed at opposite side of the stroke, where the bone at opposite side of stroke locates inside the head and supported rigidly and deflection is considered to be small by structural reason. On the other hand the occipital stroke in case (3), the high stress distribution around the head is observed as a closed loop and at the opposite side strong intensity of negative pressure is observed. This is caused by the deflection of skull at the opposite side of stroke. In both case, the stress propagation inside the brain seems not to play important role. Willinger et al [11,12] reported the result based on vibration analysis and possibility was indicated that the lesion mechanisms should be distinguished as a function of the spectral characteristics of the shock. The mechanism linked to skull deformations, the shock with durations below $4 \mathrm{msec}$ should be considered, and the mechanism linked to intracerebral stress conditions and intracerebral deformations the shock durations more than $10-12 \mathrm{msec}$ should be considered. In this study, as the duration of input loading is shorter than $10 \mathrm{msec}$ skull deformation seems to be dominant.

\section{Summary and conclusions}

The mechanism of cerebral contusion has been investigated by Finite Element Method. In order to verify the numerical analysis, the experimental study was carried out by using a water-filled acrylic cylindrical container. Tied and slide interface conditions between container wall and water were tried and both results were compared with experimental result. Both results showed rather good agreement with experiment. Tied condition showed slightly higher amplitude in 
pressure fluctuation and sliding condition seemed to give higher damping coefficient. Negative pressure appeared suddenly at the opposite side of stroke when the first peak of flexural vibration of container wall coming from both sides met just the opposite point of the stroke, and during stress wave propagation in container wall, big pressure fluctuation were not observed inside the water. The positive pressure caused at the stroke point faded away without transmitting at the opposite side. And the frequency of pressure fluctuation was close to the natural frequency of a water-filled acrylic container. After verification of numerical analysis by experiment with a simple physical model, the numerical calculation was carried out for a three-dimensional finite element human head model which consists of skull, CSF and brain. The first the result was verified by comparing with the Nahum's experiment with cadaver subjects, and showed good agreement. Next impact loadings were applied to other five parts of the head and pressure response of coup contusion and contrecoup contusion were calculated. In parietal and temporal stroke, the amplitude of negative pressure of contrecoup side was smaller than coup side, and other three cases, both amplitudes were almost the same.

\section{References}

[1] Adams, J.H., Scott, G., Parker, L.S., Graham, D.I. \& Doyle, D., The contusion index: A quantitative approach to cerebral contusions in head injury, Neuropathology and Applied Neurobiology, 6, pp.319-324, 1980.

[2] Fujiwara, S., Yanagida, Y. \& Mizoi, Y., Impact-Induced intracranial pressure caused by an accelerated motion of the head or by skull deformation; an experimental study using physical models of the head and neck, and ones of the skull, Forensic Science International, 43, pp.159-169, 1989.

[3] Yanagida, Y., Fujiwara, S. \& Mizoi, Y., Differences in the intracranial pressure caused by a 'blow' and/or a 'fall'-An experimental study using physical models of the head and neck, Forensic Science International, 41, pp.135-145, 1989.

[4] Zhang, L., et al., Recent advances in brain injury research: A new model and new experimental data, BED-50, Bioengineering Conf. pp.833-834, 2001

[5] Hosey, R.R. \& Liu, Y.K., A homeomorphic finite element model of the human head and neck, Finite Element in Biomechanics, Chapter 18, pp.379-401, 1982.

[6] Huang, H.M. et al., Finite element analysis of brain contusion: an indirect impact study, Medical \& Biological Engineering \& Computing, 38, pp.253-259, 2000.

[7] Ruan, J.S., Khalil, T. \& King, A.I., Dynamic response of the human head to impact by three-dimensional finite element analysis, Journal of Biomechanical Engineering, 116, pp.44-50, 1994.

[8] Kenner, V.H. \& Goldsmith, W., Impact on a simple physical model of the head, J. Biomechanics, 6, pp.1-11, 1973. 
[9] Nahum, A.M. \& Smith, R., Intracranial pressure dynamics during head impact, 21st Stapp car Crash Conference, pp.339-366, 1977.

[10] Galford, J.E. \& McElhaney, J.H., A Viscoelastic Study of Scalp, Brain and Dura, Journal of Biomechanics, 3, pp.211-221, 1970.

[11] Willinger, R., Kopp, C.M. \& Cesari, D., Cerebral motion and head tolerance, 35th Proceedings of the Association for the Advancement of Automotive Medicine(ISSN), Toront, pp.387-404, 1991.

[12] Willinger, R., Taleb, L. \& Kopp, C-M., Modal and Temporal Analysis of head Mathematical models, Journal of Neurotrauma, 12(4), pp.743-754, 1995. 